\title{
riccafd
}

Revista Iberoamericana de Ciencias de la Actividad Física y el Deporte

\section{GAMIFICACIÓN EN EDUCACIÓN FÍSICA: UN ANÁLISIS SISTEMÁTICO DE FUENTES DOCUMENTALES}

\section{GAMIFICATION IN PHYSICAL EDUCATION: A SYSTEMATIC ANALYSIS OF DOCUMENTARY SOURCES}

\author{
León-Díaz,Óscar ${ }^{1}$, Martínez-Muñoz, Luis Fernando ${ }^{2}$ y Santos-Pastor, María Luisa ${ }^{3}$ \\ ${ }^{1}$ León-Díaz,Óscar. Doctorando en Ciencias de la Actividad Física y el Deporte, Departamento \\ de Educación Física, Deporte y Motricidad Humana en la Universidad Autónoma de Madrid \\ (España), oscar.leon@predoc.uam.es \\ ${ }^{2}$ Martínez-Muñoz, Luis Fernando. Profesor, Departamento de Educación Física, Deporte y \\ Motricidad Humana en la Universidad Autónoma de Madrid (España), f.martinez@uam.es \\ ${ }^{3}$ Santos-Pastor, María Luisa. Profesora, Departamento de Educación Física, Deporte y \\ Motricidad Humana en la Universidad Autónoma de Madrid (España), marisa.santos@uam.es
}

Contacto: María Luisa Santos Pastor. marisa.santos@uam.es

DOI: http://dx.doi.org/10.24310/riccafd.2019.v8i1.5791

\section{RESUMEN}

Una Educación Física de calidad debe ofrecer experiencias motrices auténticas, valiosas y relevantes para la vida de las personas. Esto exige que los procesos de enseñanza aprendizaje sean progresivos y significativos, con una orientación formativa clara, apoyados en estrategias metodológicas de carácter lúdico. Es necesario que estos procesos estén conectados con las necesidades actuales de los jóvenes y que conlleve la creación de un bagaje motriz amplio y diverso, transferible a su vida cotidiana y con efectos positivos sobre su salud. Conseguir estos retos exige revisar los procedimientos con los que se enseña y aprende. El presente artículo se centra en revisar el estado de la cuestión sobre las estrategias de aprendizaje en Educación Física, en particular sobre la gamificación, con la finalidad de comprender su significado y sus posibles aplicaciones en la EF escolar, así como la repercusión en el proceso de enseñanza-aprendizaje. Para alcanzar este objetivo se ha procedido a hacer un análisis bibliométrico sobre el estado de la cuestión, mediante la revisión de diferentes bases de datos de acceso restringido y de libre acceso. Los resultados muestran la consideración de nuevas metodologías para la enseñanza de la Educación Física, en las que el juego aparece como un eje de interés en todos los ámbitos educativos. El estudio realizado revela la emergencia de nuevas o "reformadas" estrategias metodológicas lúdico-motrices como es la de gamificación, siendo considerada como una potente herramienta para aprender competencias.

Palabras clave: gamificación, metodologías activas, modelos de enseñanza, investigación documental, análisis de contenido. 


\section{ABSTRACT}

A quality Physical Education must offer authentic, valuable and relevant motor experiences for people's lives. This requires that the teaching-learning processes be progressive and significant, with a clear formative orientation, supported by methodological strategies of a playful nature. It is necessary that these processes are connected to the current needs of young people and that it entails the creation of a broad and diverse motor baggage, transferable to their daily life and with positive effects on their health. Achieving these challenges requires reviewing the procedures with which you teach and learn. This article focuses on reviewing the state of the question on learning strategies in Physical Education, in particular on gamification, in order to understand their meaning and possible applications in school PE, as well as the impact on the process teaching-learning. In order to achieve this objective, a bibliometric analysis was carried out on the state of the matter, through the revision of different databases of restricted access and free access. The results show the consideration of new methodologies for the teaching of Physical Education, in which the game appears as an axis of interest in all educational areas. The study reveals the emergence of new or "reformed" ludic-motor methodological strategies such as gamification, being considered as a powerful tool for learning competencies.

Key words: gamification, active methodologies, teaching models, documentary research, content analysis.

\section{INTRODUCCIÓN}

Las metodologías educativas están cambiando para dar prioridad a la amplitud y profundidad del aprendizaje, tal y como señalan los dominios de aprendizaje de la Comisión Especial sobre Métricas de los Aprendizajes (LMTF, 2013). La Educación Física (EF), para poder ser considerada de calidad, debe brindar distintas oportunidades y experiencias de aprendizaje debidamente organizadas y sistematizadas, con un objetivo claramente educativo, que transmita valores y desarrolle competencias. Es necesario que la EF que se enseña en las escuelas ofrezca un currículo adaptado a las necesidades de los jóvenes y acorde con las transformaciones sociales, que dote a los jóvenes de habilidades motrices, comprensión cognitiva y aptitudes sociales y emocionales que necesitan para llevar una vida físicamente activa (McLennan y Thompson, 2015).

En este marco el juego constituye una herramienta fundamental para lograr aprendizajes valiosos y útiles para poder aplicar a la vida. De hecho, el juego ha sido un objeto de estudio muy presente en la educación, en general yen la EF, en particular. Diferentes autores de ámbitos científicos diversos (filósofos, psicólogos, pedagogos) como Caillois, Cagigal, Piaget, Huizinga, Tonucci..., coinciden en señalar su importancia como elemento con un gran potencial educativo. Actualmente, en las sociedades tecnológicas, el juego adquiere nuevos significados, siendo impulsado desde diferentes ámbitos y adquiriendo nuevos significados. 
En concreto, surge un nuevo concepto como es el de gamificación, que trata de integrar las dinámicas y mecánicas de los videojuegos a través de cualquier soporte, digital o no, con el objetivo de aumentar la participación, motivar al alumnado y desarrollar competencias basadas en el aprendizaje, para trabajar los contenidos educativos como si fuera un juego (Durall, Gros, Maina, Johnson y Adams, 2012).

El término gamificación es bastante reciente. Según Deterding, O'Hara, Sicart, Dixon y Nacke (2011) no se adoptó ampliamente antes de la segunda mitad de 2010. Kapp (2012) señala que se trata de utilizar las mecánicas del juego, su estética y sus estrategias para involucrar a la gente, motivar la acción, promover el aprendizaje y resolver problemas. Todo ello con el objetivo de modificar o promover comportamientos deseados (Lee y Hammer, 2011). Zichermann y Cunningham (2011) mencionan que la gamificación puede ser entendida como el uso de elementos de los sistemas de juegos con objetivos mercantiles. En este sentido, parece que siendo usada para crear experiencias que emplean el poder de los videojuegos para aplicarlo en campos diversos como: salud, finanzas, gobernación, educación, etc.

En el contexto educativo, el Informe Horizon (2014), propone la gamificación como una tendencia educativa válida para contextos universitarios debido al auge de los juegos y aplicaciones en los dispositivos móviles. En esta línea, el Informe INNEDU (2016) corrobora que este tipo de planteamientos e intervenciones didácticas están consolidándose en los entornos educativos españoles como nueva metodología activa, con el propósito de mejorar la adherencia al aprendizaje e incrementar la motivación entre el alumnado.

Las revisiones de literatura sobre gamificación son, relativamente, escasas (Hamary, Koivisto y Sarsa, 2014; Dicheva, Dichev, Agre y Angelova, 2015; Pascuas, Vargas y Muñoz, 2017; González, Del Río y Navarro, 2018) y sólo una de ellas está enfocada al ámbito educativo. En esta comunicación, se presenta una revisión sistemática de documentos científicos de EF dedicados a la gamificación. El estudio nos permite describir e interpretar la realidad con el propósito de poder comprender su significado y sus posibles aplicaciones en la EF escolar, así como la repercusión en el proceso de enseñanza-aprendizaje. Para ello, trataremos de responder los siguientes interrogantes: ¿Qué documentación ha sido publicada que haga referencia a la gamificación en EF? ¿Cómo se ha conceptualizado la estrategia en nuestro ámbito? ¿Cómo incide la aplicación de la gamificación en los procesos de enseñanza y aprendizaje? ¿Debemos poner en valor la gamificación como metodología activa dentro del contexto de la EF?

\section{MATERIAL Y MÉTODOS}

Estrategia de búsqueda: el acceso a las fuentes documentales publicadas por diferentes autores, tanto en España como en el contexto internacional, ha sido realizado durante los meses de agosto y septiembre del 2018 a través de las 
siguientes bases de datos acreditadas en el campo de las Ciencias Sociales: bases de datos de libre acceso (Google scholar, Redalyc y Dialnet) y bases de datos con acceso restringido (ISI Web of Knowledge, Scopus y EBSCO). Dada la variabilidad terminológica existente sobre el tema, ha sido necesario revisar algunas fuentes documentales (Dicheva et al., 2015) para contrastar opiniones y definir cuál podrían ser los descriptores más valiosos para hacer la búsqueda. Finalmente, hemos utilizado como términos de búsqueda las palabras "educación física", "gamificación", "physical education" y "gamification", utilizando los conectores Y-AND segun el idioma de las palabras utilizadas. Esta búsqueda no se limitó por año de publicación aunque se introdujo como límite el idioma utilizado (español e inglés).

Criterios de inclusión y exclusión: en la búsqueda de publicaciones se incluyó todo tipo de documentos pertenecientes al campo de la EF que estuvieran relacionadas con la gamificación. Respecto a las publicaciones encontrados en las bases de datos científicas se aplicó como criterio de inclusión el que las publicaciones fueran textos originales, tuvieran un formato de texto completo y hubieran sido publicados en una revista científica, o en su defecto, que hayan sido publicados, en actas de congresos internacionales de EF.

Extracción de datos: tras la búsqueda inicial, se localizaron 483 publicaciones, aunque se excluyeron 435 que no fueron relevantes para el objetivo de esta revisión, por no cumplir con los criterios definidos para este estudio. Para proceder a la selección, se revisaron los abstracts y, en caso necesario, los artículos completos, con el fin de decidir si la información que contenían estaba o no relacionada con nuestro objetivo. Finalmente, se seleccionaron 26 publicaciones, (revistas científicas y actas de congresos) ${ }^{1}$.

Análisis de los datos: a partir de la literatura especializada revisada y la consulta a expertos en el tema, se llevó a cabo un análisis de contenido (Andreu, 200) desde el establecimiento de cuatro focos de interés o temáticos, vinculados con cada uno de los interrogantes planteados en el estudio (Ver Tabla 1). Las categorías de análisis surgen de manera deductiva de la revisión previa del marco teórico, empleando software informáticos como el "Mendeley" y el "NVIVO 12", para realizar el análisis de la información.

\section{RESULTADOS}

Los resultados del estudio para analizarlos con más detalle sus características, se presentan atendiendo a cuatro dimensiones: tipológica, práctica, teórica y crítica. En la tabla 1 se muestra la composición de cada una de las dimensiones establecidas según el sistema de codificación efectuado en el análisis de contenido.

\footnotetext{
${ }^{1}$ Se comparte un anexo donde se detallan las publicaciones revisadas mediante este enlace: https://drive.google.com/open?id=1HA3Kv5NfMzXXfOSLGtthKQoXRTBa97mM.
} 
Tabla 1: Sistema de codificación/categorización del análisis de contenido.

\begin{tabular}{|c|c|}
\hline Dimensión tipológica & Dimensión práctica o curricular \\
\hline $\begin{array}{l}\text { ¿Qué documentación ha sido publicada que } \\
\text { haga referencia a la gamificación en EF? }\end{array}$ & $\begin{array}{l}\text { ¿Cómo se ha conceptualizado la estrategia } \\
\text { en nuestro ámbito? }\end{array}$ \\
\hline Año de publicación & Competencias \\
\hline Etapa educativa & Contenidos \\
\hline \multirow[t]{3}{*}{ Tipología de la publicación: } & Interdisciplinariedad \\
\hline & Hibridación metodológica \\
\hline & Evaluación \\
\hline Dimensión teórica & Dimensión crítica \\
\hline $\begin{array}{l}\text { ¿Cómo incide la aplicación de la gamificación en } \\
\text { los procesos de enseñanza y aprendizaje? }\end{array}$ & $\begin{array}{l}\text { ¿Debemos poner en valor la gamificación } \\
\text { como metodología activa dentro del } \\
\text { contexto de la EF? }\end{array}$ \\
\hline Definición & Objetivos, finalidades y propósitos \\
\hline Características & Principales hallazgos \\
\hline Aspectos claves & Cuestionamientos \\
\hline \multicolumn{2}{|l|}{ Teorías relacionadas } \\
\hline Conceptos relacionados & \\
\hline
\end{tabular}

\section{Dimensión tipológica}

Los estudios sobre gamificación en EF en España son bastante recientes. El primer trabajo publicado (Monguillot, González, Zurita, Almirall y Guitert, 2015) nos presenta una unidad didáctica para $2^{\circ} \mathrm{ESO}$ para el desarrollo de conductas saludables. En la figura 1, recopilamos y clasificamos los artículos encontrados sobre gamificación, diferenciándolos según su tipología en: artículos de investigación, de reflexión y de aplicación didáctica.

Asimismo, podemos observar que las referencias a la gamificación en EF se extienden a los diferentes niveles educativos (Ver figura 2), siendo más numerosos los de Educación Primaria, seguidos de Educación Secundaria y Bachillerato y Educación Superior, siendo la etapa de Educación Infantil la que menor relevancia adquiere, con ninguna referencia localizada. En este análisis cabe destacar las publicaciones de Morente, Romance, Gil y Benítez (2018); Guerrero (2017); Martínez, De la Torre y Ruiz (2017); Fernández (2018); y Trillo, Palomares, González y De las Heras (2018), quienes combinan las etapas educativas de Primaria y Secundaria. También destaca la publicación de Alcaraz, Sánchez y Grimalda (2018), que combinan las etapas educativas de Secundaria y Educación Superior.

\section{Dimensión práctica}

El mayor número de artículos encontrados pertenecen a la tipología de"aplicación didáctica" por lo que hemos analizado con más detalle las características compren- 


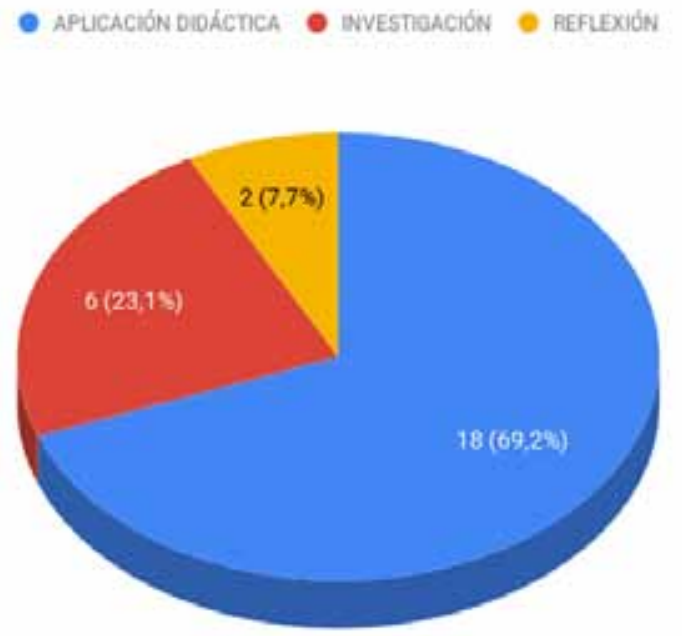

Figura 1. Relación de artículos publicados en función de su tipología. Elaboración propia.

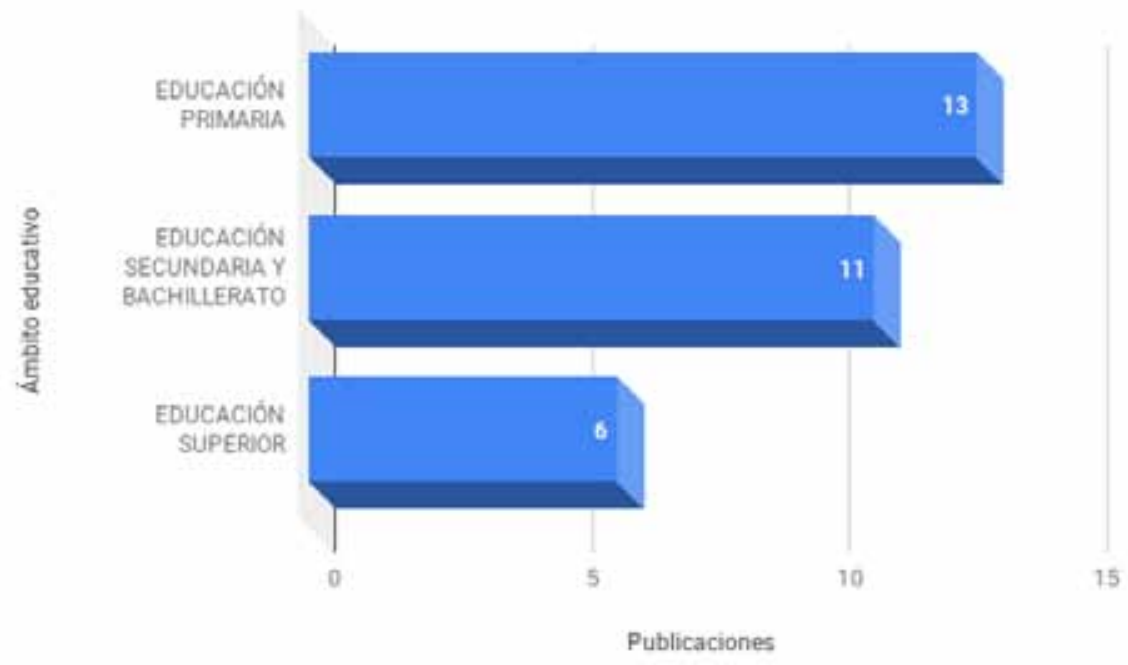

Figura 2. Etapas educativos de investigación y aplicación didáctica de la Gamificación en EF. Elaboración propia.

didas desde una dimensión práctica-curricular, destacando: (1) competencias (2) contenidos (3) interdisciplinariedad; (4) hibridación metodológica; y (5) evaluación. En la tabla 2 se muestran los resultados obtenidos. No obstante, cabe indicar que solo se han incluido las publicaciones que hacen referencia, de forma explícita o implícita, a los elementos curriculares que se exponen en las experiencias prácticas.

\section{Dimensión teórica}

Entre las publicaciones analizadas encontramos algunas que desarrollan teorías relacionadas con la gamificación. En algunas de ellas se reconoce el juego como factor cultural y social en cualquier actividad, siendo Huizinga y Callois los 
Tabla 2. Elementos curriculares desarrollados a través de la gamificación en EF.

\section{COMPETENCIAS}

Comunicación lingüística: Martínez et al. (2017) y Navarro et al. (2017)

Matemática, ciencia y tecnología: Alcaraz et al. (2018); Díaz y Requena (2016); Hortigüela et al. (2018); Monguillot et al.(2015); Morente et al. (2018) y Navarro et al. (2017)

Digital: Alcaraz et al.(2018); Díaz y Requena (2016); González (2018); Martínez (2017); Martínez et al. (2017); Monguillot et al. (2015); Morente et al. (2018); Ortí (2018); Quintero et al (2018) y Trillo et al. (2018)

Aprender a aprender: Alcaraz et al. (2018); Almirall (2016); Fernández et al. (2018); Flores y Prat (2018); Martínez et al.(2017); Morente et al. (2018) y Navarro et al. (2017)

Sentido de iniciativa y espíritu emprendedor: Díaz y Requena (2016) y Navarro et al. (2017)

Conciencia y expresiones culturales: Díaz y Requena (2016); Flores y Prat (2018); Pérez et al. (2017) y Quintero et al. (2018)

Sociales y cívicas: Alcaraz et al. (2018); Almirall (2016); Fernández (2018); Flores y Prat (2018); González (2018); Hortigüela et al. (2018); Martínez (2017); Martínez et al. (2017); Monguillot et al. (2015); Navarro et al. (2017) y Trillo et al. (2018)

\section{INTERDISCIPLINARIEDAD}

Educación artística, Lengua, Ciencias Naturales y Música: Fernández et al. (2018)

Ciencias Sociales: Díaz y Requena (2016) y Fernández et al. (2018)

Matemáticas: Hortigüela et al.(2018) y Morente et al. (2018)

\section{CONTENIDOS}

Acciones motrices individuales en entornos estables: Flores y Prat (2018); González (2018); Monguillot et al. (2015); Morente et al. (2018); Navarro et al. (2017); Ortí (2018); Pérez et al. (2017) y Trillo et al. (2018)

Acciones motrices en situaciones de oposición: Almirall (2016); Flores y Prat (2018); Guerrero (2017); González (2018); Navarro et al. (2017); Pérez et al. (2017); Quintero et al. (2018) y Trillo et al. (2018)

Acciones motrices en situaciones de cooperación, con o sin oposición: Fernández (2018); Flores y Prat (2018); González (2018); Hortigüela et al. (2018); Martínez et al. (2017); Monguillot et al. (2015); Navarro et al. (2017); Pérez et al. (2017): Quintero et al. (2018) y Trillo et al. (2018)

Acciones motrices en situaciones de adaptación al entorno físico: Alcaraz et al. (2018); Díaz y Requena (2016); Fernández et al. (2018); Flores y Prat (2018); González (2018); Navarro et al. (2017); Pérez et al. (2017) y Quintero et al.(2018)

Acciones motrices en situaciones de índole artística o de expresión: Díaz y Requena (2016); Flores y Prat (2018); González (2018); Navarro et al. (2017); Pérez et al. (2017) y Quintero et al. (2018)

\section{HIBRIDACIÓN METODOLÓGICA}

Aprendizaje cooperativo: Almirall (2016); Díaz y Requena (2016); Flores y Prat (2018); Fernández (2018); González (2018); Hortigüela et al (2018); Martínez (2017); Martínez et al. (2017); Monguillot et al. (2015); Morente et al. (2018); Navarro et al. (2017); Ortí (2018); Pérez et al. (2017) y Quintero et al. (2018)

Resolución de problemas y descubrimiento guiado. Monguillot et al. (2015) y Navarro et al. (2017)

Pedagogía de la aventura: Fernández et al.(2018)

Otras (sport education, clase invertida, aprendizaje por proyectos, aprendizaje servicio): González (2018) y Navarro et al. (2017) 


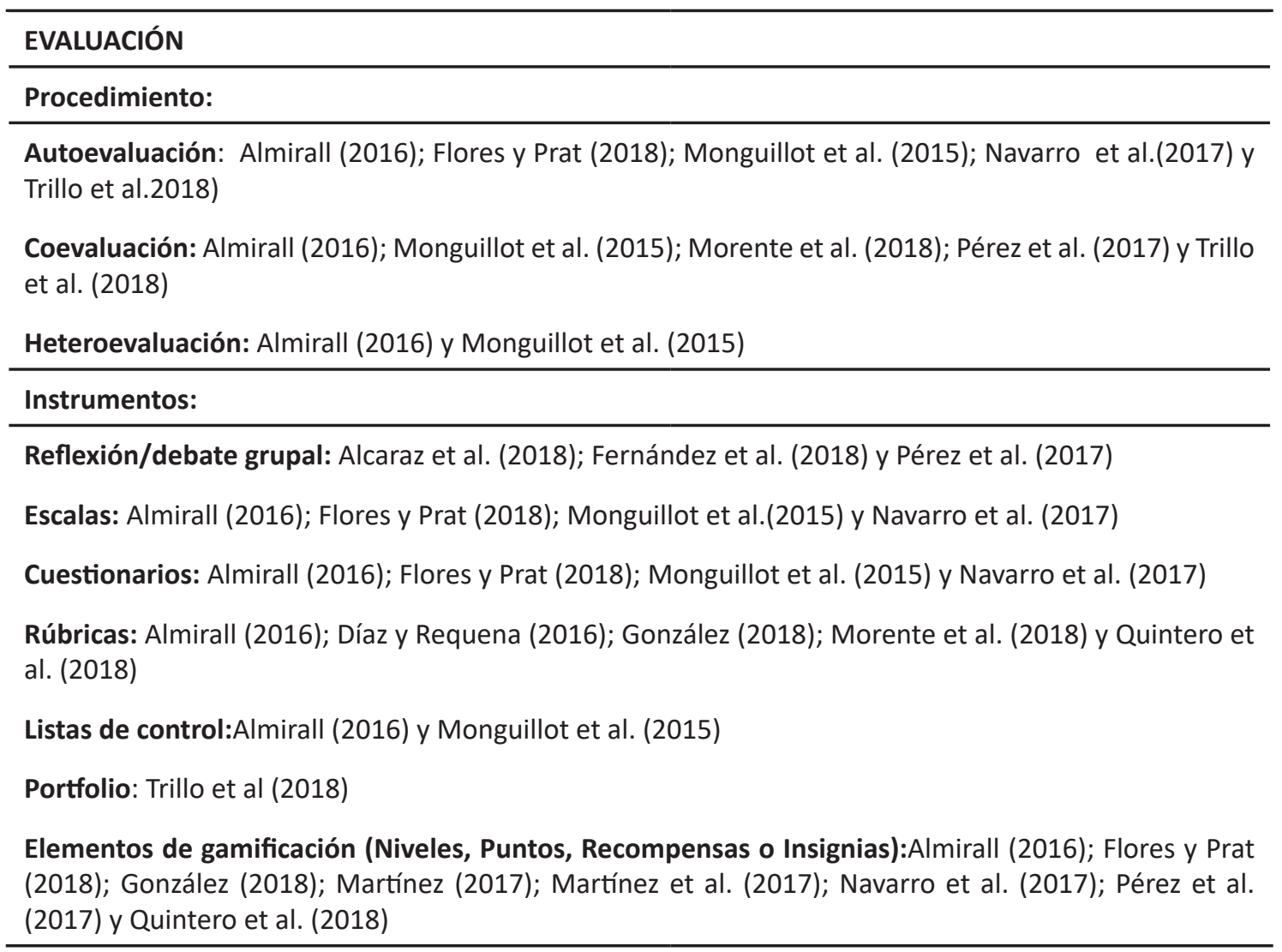

principales propulsores de esta idea (Brasó, 2018). Por su parte, algunos autores hacen referencia a la teoría social del aprendizaje de Bandura, quien argumenta el valor de la autoeficacia y capacidad del individuo para lograr un objetivo que puede verse alterado por las experiencias de éxito, indirectas o la persuasión social, tal y como suele ocurrir en los entornos gamificados (Kostenius, Hallberg y Lindqvist, 2018). Asimismo, los avances en el campo de la Neurociencia, ensalzan el valor del juego como generador de dopamina y emociones, y por tanto propulsores del aprendizaje (Navarro, Martínez y Pérez, 2017).

El concepto gamificación ha sido definido desde diferentes concepciones. En primer lugar, como un planteamiento pedagógico que consiste básicamente en el uso de elementos propios de los juegos en contextos donde no hay juego (Fernández, 2018). En segundo lugar, como una metodología donde el profesorado elabora un conjunto de actividades de aprendizaje en base a una historia o narrativa, que incluye un conjunto de elementos del juego (Flores y Prat, 2018), que integra las dinámicas de juego a través de cualquier soporte, digital o no (Gómez, Molina y Devis, 2018), cuyo objetivo principal es involucrar al alumnado en una aventura lúdica, vinculada estrechamente con su aprendizaje (Quintero, Jiménez y Area, 2018). En tercer lugar, como una técnica, un método y una estrategia a la vez que consiste en introducir estrategias y dinámicas propias de los videojuegos en contextos ajenos o no lúdicos (Monguillot et al., 2015). 
Por otra parte, podemos resaltar la aparición de otros conceptos que se han ido añadiendo a la gamificación referida al ámbito educativo. Encontramos términos como Escape Room (Fernández, 2018), Exergame (González, Gómez, Navarro, Cairós, Quirce, Toledo y Marrero, 2016), Juegos de rol (Fernández, Prieto, Alcaraz, Sánchez y Grimaldi, 2018) o juegos serios (Kostenius et al., 2018), que aunque con matices diferentes, el objetivo fundamental de todos ellos, como en todo entorno educativo gamificado, trata de adaptar las aulas para crear contextos de aprendizaje creativos basados en el juego. Del mismo modo, también se destaca la diferenciación entre la gamificación con respecto al aprendizaje basado en juegos, donde los juegos ya existen y cuyas mecánicas ya están establecidas (González, 2018).

En relación con los elementos del juego introducidos en las diferentes aplicaciones didácticas analizadas, destacamos posibilidades diversas de ambientación o narrativas empleadas como: creación de mundos o aldeas (Navarro et al., 2017), escenarios de fantasía (Alcaraz, Sánchez y Grimaldi, 2018), adaptadas de películas (González, 2018), basadas en videojuegos (Miriam, 2017), espacios imaginados o inspirados en situaciones de la vida real (Fernández, 2018), entre otros. También, se menciona el empleo de diferentes tipos de retos, ya sean individuales (Quintero et al., 2018), cooperativos (Fernández, 2018), competenciales (Navarro et al., 2017); o bien, relacionados con las habilidades motrices básicas (Fernández et al., 2018), así como la agrupación del alumnado en equipos o grupos: por especialidad (Pérez, Rivera y Trigueros, 2017), heterogéneos (Trillo et al., 2018), por sus conocimientos iniciales (Quintero et al., 2018), aleatorios (Martínez et al., 2017), entre otras divisiones. Por último, cabe destacar el uso de elementos del juego como: misiones (González, 2018), recompensas (Navarro et al., 2017), insignias (Guerrero, 2017), clasificaciones (Flores y Prat, 2018), niveles (Pérez et al., 2017) y poderes (Quintero et al., 2018).

En relación con aspectos clave para llevar a cabo proyectos gamificados, aparecen varios términos ligados a este hecho, como el de diversión, ya que el juego es en sí mismo es un elemento motivador (Brasó, 2018). Además, para que la gamificación tenga éxito en el contexto educativo, la propuesta tiene que partir de la voluntariedad (Pérez et al., 2017), debe ser interesante y flexible, de tal manera que comprometa e implique al alumnado en el proceso (Monguillot et al., 2015). Desde este punto de vista, su uso debe basarse en la posibilidad de autoevaluar los resultados y corregir los errores de forma que se cree un ciclo de aprendizaje mediante la búsqueda de soluciones y evaluación de resultados (Gómez et al., 2018). También, en la actualidad, el elemento tecnológico está muy relacionado con la gamificación en la mayoría de los proyectos analizados, aunque en mayor o menor medida, no es determinante (Navarro et al., 2017). Para considerar que un entorno con carácter didáctico esté gamificado se deben planificar todos los elementos curriculares dentro de su propia estructura, partiendo de la premisa de convertir los objetivos de materia en objetivos de nuestras misiones (González, 2018) o retos (Quintero et al., 2018). En este sentido, el profesorado debe contextualizar el uso de la gamificación dentro de su programación educativa, con unos objetivos pedagógicos concretos y aprovechan- 
do su potencial experimental para complementar las necesidades pedagógicas del profesorado y del alumnado (Gómez et al., 2018). Desde esta perspectiva, se trata de transmitir al alumnado la importancia de vivir esta propuesta no como una asignatura finita, donde la meta es ella misma, sino como una experiencia de aprendizaje que realmente le enriquezca y favorezca su proceso formativo (Pérez et al., 2017). Por último, de forma específica en el área, el docente que quiera desarrollar entornos gamificados en EF debe tener muy en cuenta el nivel de actividad física que implica la realización de la propuesta, ya que es uno de los objetivos que sólo puede conseguirse desde nuestro área, proporcionando la unión necesaria entre el entorno gamificado y el ámbito motor de la Educación Física (Fernández, 2018), además de poder contribuir a cambiar la percepción de las familias con respecto a la materia (González, 2018).

\section{Dimensión crítica}

En la revisión realizada observamos que la gamificación emerge en el contexto de la EF desde diversas perspectivas, estrechamente relacionadas. Por un lado, existe una visión centrada en el alumnado, que trata de: (1) promover y desarrollar estilos de vida saludables (Monguillot et al., 2015); (2) adquirir valores personales (Trillo et al., 2018); (3) alcanzar un aprendizaje emocionalmente significativo, (Navarro et al., 2017); (4) conseguir una mayor implicación del alumnado (Guerrero, 2017); y, (5) acercando al aula la realidad que este vive en su día a día (Hortigüela, Hernándo y Pérez, 2018) conectando con sus intereses y pasiones (Almirall, 2016). Por otro lado, desde un horizonte centrado en el profesorado, la gamificación trata de: (1) alcanzar los objetivos de materia y competencias clave en EF (Quintero et al., 2018); (2) desarrollar una serie de contenidos curriculares (Marín y Sampedro, 2016); (3) incidir en el desarrollo de competencias profesionales (Pérez y Rivera, 2017) que le ayuden a mejorar la labor docente (González, 2018) e incluso, (4) a ser mejores personas (Trillo et al., 2018).

Los hallazgos dilucidados evidencian dos grandes temáticas predominantes: motivación y aprendizaje. Respecto a la motivación, la gamificación ha conseguido mejorar la motivación del alumnado, tanto por la EF como por la adhesión a la práctica de actividad física (Navarro et al., 2017), siendo en menor medida en la etapa de Secundaria (Trillo et al., 2018). No obstante, es necesario que el docente esté involucrado para encauzar y mantener la motivación durante toda la duración de la propuesta (Alcaraz et al., 2018), pues se ha observado que, con el paso del tiempo, el impacto inicial se diluye, hasta tal punto de que una gran parte del alumnado no se siente atraído por sus dinámicas y mecánicas (Flores y Prat, 2018). Por lo que se refiere al aprendizaje, se ha reflejado como aumenta el compromiso y el rendimiento del alumnado por la práctica física y el desarrollo de conductas saludables (Almiral, 2018). Sin embargo, en cuanto a la mejora de los aprendizajes en contenidos concretos, el alumnado se muestra prudente en relación a sus avances (Quintero et al.,2018) y experimenta dificultades, tales como reconocer que su papel es primordial para su aprendizaje (Pérez, Rivera y Delgado, 2017). Al mismo tiempo, se pone de manifiesto que el trabajo cooperativo se ve impulsado por este tipo de experiencias, mostrándose sensible a las 
conductas prosociales de tolerancia, colaboración y respeto (Fernández, 2018). También, se evidencia que la gamificación facilita la creación de un clima de aula distendido (Navarro et al., 2017), disminuyendo las conductas disruptivas (Trillo et al., 2018) y generando una sensación de control y asunción de responsabilidad, que les acerca a la oportunidad de poder construir sus propios aprendizajes (Pérez et al., 2017). Por último, desde el punto de vista del profesorado, se valora las diversas posibilidades para atender los diferentes ritmos de aprendizaje del alumnado (Monguillot et al., 2015).

Siguiendo con el análisis crítico, algunos autores han reflexionado sobre las propuestas de gamificación, cuestionado su presencia e implementación. En el ámbito educativo, se han añadido metodologías que disponen de unos aspectos potenciadores de autonomía, pero otros que se basan en aspectos encaminados para adaptar la juventud a la sociedad (Brasó, 2018). Así pues, gamificar en EF puede parecer sorprendente y hasta parecer innecesario, en un área que siempre ha estado vinculada al juego y a las actividades lúdicas, al menos de manera teórica (Fernández, 2018). Cuando "gamificamos" creamos específicamente un entorno para alcanzar nuestros objetivos de materia, no debemos asumir que cualquier situación jugada está gamificada (Quintero et al., 2018). Por otro lado, cabe destacar la percepción negativa que existe por parte del profesorado hacia las posibilidades educativas del videojuego, así como una falta de conocimientos de programación necesarios para su implementación (Gómez et al., 2018), que requieren de un esfuerzo extra por parte del profesorado (Trillo et al, 2018). Igualmente, no parece fácil cambiar el pensamiento del alumnado referente a la tradicional forma de aprender (Navarro et al., 2017). De hecho, a veces, la emoción y el ansia por el logro le hace olvidar la esencia pedagógica de las propuestas de EF (Hortigüela, Hernando y Pérez, 2018). Por último, en las mecánicas y dinámicas del juego, dependiendo del enfoque que adquiera, hace que el alumnado se muestre muy competitivo, de modo que su deseo de ganar al sistema da lugar, en muchas ocasiones, a resultados de aprendizaje poco deseables (lb.).

\section{DISCUSIÓN}

Esta comunicación ha presentado una revisión sistemática de documentos científicos de EF dedicados a la gamificación. El estudio nos ha permitido describir e interpretar la realidad con el propósito de poder responder a alguno de los interrogantes que nos planteamos inicialmente:

\section{¿Qué documentación ha sido publicada que haga referencia a la gamificación en EF?}

La producción científica revisada se basa, mayoritariamente, en la descripción y comunicación de experiencias y aplicaciones didácticas, que generalmente se circunscriben a la etapa de Primaria y Secundaria. Por lo tanto, de acuerdo con Dicheva et al. (2015), se necesita una investigación empírica más sustancial. En concreto, se precisa de estudios que reflexionen sobre los efectos motivadores 
del uso de los distintos elementos del juego para producir aprendizajes propios de EF. Esto permitiría al profesorado tener mayor conocimiento sobre los efectos beneficiosos de la gamificación y les ayudaría a decidir qué elementos del juego utilizar en su contexto específico para conseguir con mayores garantías de éxito los aprendizajes deseados.

\section{¿Cómo se ha conceptualizado la estrategia en nuestro ámbito?}

En resumen, y en consonancia con la mayoría de los autores, la gamificación en la EF es entendida como el empleo de mecánicas y dinámicas propias de los juegos con la finalidad de motivar al alumnado durante su proceso de aprendizaje. No obstante, existe cierta controversia, a la hora de integrar este concepto como una pedagogía, metodología, técnica, estrategia, recurso o todas ellas al mismo tiempo. A nuestro parecer, en base al análisis realizado, la gamificación no deja de ser una estrategia metodológica que diseña, desarrolla y aplica actividades concretas en momentos dados para la consecución de objetivos específicos, pudiendo ser enfocada desde diferentes creencias y estilos de enseñanza.

\section{¿Cómo incide la aplicación de la gamificación en los procesos de enseñanza y aprendizaje en EF?}

Desde el estudio de la dimensión práctica, hemos podido comprobar que, la creación de un entorno gamificado, puede integrar todos y cada uno de los elementos curriculares establecidos en el marco legislativo. La gamificación es adecuada para un enfoque competencial, es capaz de trabajar todo tipo de situaciones motrices o contenidos, puede combinarse e hibridarse con diferentes modelos pedagógicos o estilos de enseñanza, y es capaz de valorar los aprendizajes producidos desde diversos procedimientos e instrumentos de evaluación. Concretamente, hemos evidenciado una mayor presencia de entornos gamificados para la adquisición de la competencia digital y la competencia social y cívica, que se trabajan a través de situaciones motrices de colaboración. Asimismo, vemos que toman como referencia un modelo pedagógico de aprendizaje cooperativo y entiende la evaluación como parte del proceso, utilizando las propias mecánicas y dinámicas del juego para su desarrollo. Por lo tanto, consideramos que la gamificación es capaz de incidir en el proceso de enseñanza-aprendizaje en EF, creando un entorno de aprendizaje, lúdico, atractivo, actual, flexible y contextualizado, apto para generar experiencias de aprendizajes significativas para el alumnado.

\section{¿Debemos poner en valor la gamificación como metodología activa dentro del contexto de la EF?}

La gamificación emerge en el contexto de la EF para modificar la práctica docente tradicional con la finalidad de motivar al alumnado hacia la práctica y producir aprendizajes emocionalmente significativos. A través de esta estrategia metodológica, el profesorado logra conducir el comportamiento del alumnado potenciando la motivación, mediante la participación e interacción en un entorno 
gamificado. Esta metodología persigue un aprendizaje activo y permite al alumnado aprender de forma cooperativa y lúdica, desde el punto de vista educativo, personal y social. De ahí que, haciendo un análisis crítico, pensamos que se debería analizar el sentido de la gamificación, pues si se utiliza desde discursos de rendimiento y competición se estaría perpetuando una concepción tradicional de la EF. Por lo tanto, no está claro si el objetivo de la gamificación contribuye a un aprendizaje de calidad de la Educación Física, mejora la motivación del alumnado o ayuda a modificar la conducta del alumnado, considerando que sería necesario profundizar sobre este aspecto.

\section{CONCLUSIONES}

La bibliografía revisada hace referencia a que la gamificación en EF ha aumentado de forma exponencial en los últimos años. En consonancia, con los últimos informes (LMTF, 2013; Informe Horizon, 2014; Informe INNEDU, 2015), el estudio ha identificado nuevas metodologías educativas a través de la utilización de los juegos en todos los ámbitos educativos, valorándose como una tendencia emergente, que brinda distintas oportunidades y experiencias de aprendizaje a la EF. Además, ofrece realidades alternativas, combinando una gran variedad de teorías, definiciones y características que sustentan su implementación, así como diferentes justificaciones en base a las necesidades y/o respuestas a través de estas propuestas didácticas.

Los procesos de gamificación se concretan en el uso de herramientas, técnicas $u$ otros elementos extraídos del juego, que se emplean como complemento para lograr unos objetivos. Se estructuran en niveles de complejidad; fomentan la participación a través de misiones y/o retos; cuentan con un sistema de puntos y recompensas en forma de insignias o badges; y, muestran el ranking y posicionamiento del alumnado acorde a su progreso. La gamificación es una estrategia motivacional y una potente herramienta para movilizar conocimientos y consolidar aprendizaje.

En definitiva, podemos afirmar que los trabajos existentes sobre la gamificación en EF todavía son escasos, aunque, sin duda, constituye un campo emergente que incidirá en la enseñanza y el aprendizaje de la EF.

\section{REFERENCIAS BIBLIOGRÁFICAS}

Izada. Fundación Centro Estudios Andaluces, Universidad de Granada, 10 (2), 1-34.

Brasó, J. (2018). Pere Vergés: escuela y gamificación a comienzos del s. XX. Apunts Educación Física y Deportes, 133(3), 20-37.

Deterding, S., O'Hara, K., Sicart, M., Dixon, D. y Nacke, L. (2011). Gamification: Using Game Design Elements in Non-Gaming Contexts. Human Factors in Computing Systems, 2425-2428. 
Díaz, V. M y Requena, B. E. S. (2016). Innovando en el aula de Educación Primaria con GT 6. Innoeduca: international journal of technology and educational innovation, 2(1), 13-19.

Dicheva, D., Dichev C., Agre G. y Angelova G. (2015). Gamification in Education: A Systematic Mapping Study. Educational Technology \& Society, 18 (3), 75-88.

Durall, E., Gros, B., Maina, M., Johnson, L. y Adams, S. (2012). Perspectivas tecnológicas: educación superior en Iberoamérica 2012-2017. Austin, Texas: The New Media Consortium.

Fernández, J., Prieto, E., Alcaraz, V., Sánchez, A. J., y Grimaldi, M. (2018). Aprendizajes significativos mediante la Gamificación a partir del Juego de Rol: "Las Aldeas de la Historia". Espiral. Cuadernos del Profesorado, 11(22), 69-78.

Fernández, J. (2018). De los desafíos físicos cooperativos a las Educoop-Escape rooms. En actas del XI Congreso Internacional de Actividades Físicas Cooperativas. Avilés.

Flores, G., Prat, M (2018). "X-Vic: corre y vuela sobre los pirineos". Un proyecto gamificado y cooperativo en educación superior. En actas del XI Congreso Internacional de Actividades Físicas Cooperativas. Avilés.

Guerrero, L. (2017). El bádminton a través de la gamificación. Revista Digital Educativa de Ampe Asturias, (19), 45-50.

Gómez, F., Molina, P., y Devís, J. (2018). Los videojuegos como materiales curriculares: una aproximación a su uso en Educación Física. Retos, 34, 305-310.

González (2018). EL JEDI DE LA EDUCACIÓN FÍSICA. En actas del XI Congreso Internacional de Actividades Físicas Cooperativas. Avilés.

González C.S., Gómez N., Navarro V., Cairós M., Quirce C., Toledo P., Marrero N. (2016). Learning healthy lifestyles through active videogames, motor games and the gamification of educational activities. Comput. Hum. Behav. 2016;55:529-551.

Hamari, J., Koivisto, J., y Sarsa, H. (2014). Does gamification work? A literature review of empirical studies on gamification. En 2014 47th Hawaii international conference on system sciences (HICSS), 3025-3034). IEEE.

Hortigüela, A., Hernándo, A. y Pérez, A. (2018). EL ESCAPE ROOM: UNA PROPUESTA COOPERATIVA CON INFINIDAD DE POSIBILIDADES EN EDUCACIÓN FÍSICA. En actas del XI Congreso Internacional de Actividades Físicas Cooperativas. Avilés.

Informe Horizon (2014). Higher Education Edition. Austin, Texas, Estados Unidos: The New Media Consortium.

INNEDU. (2016). Guía práctica para el cambio educativo en España. Instituto de innovación en negocios educativos.

Kapp, K.M. (2012). The Gamification of learning and Instruction. San Francisco, CA: John Wiley.

Kostenius, Hallberg, \& Lindqvist, (2018). Gamification of health education: Schoolchildren's participation in the development of a serious game to promote health and learning. Health Education, 118(4), 354-368.

Lee, J.J., y Hammer, J. (2011). Gamification in Education: What, How, Why Bother. Academic Exchange Waterly, 15 (2). 
LMTF-Learning Metrics Task Force (2013). Hacia el Aprendizaje Universal: Un Marco Global para Medir el Aprendizaje. Informe $n^{\circ} 2$ de la Comisión especial sobre Métricas de los Aprendizajes. Montreal y Washington: Instituto de Estadística de la UNESCO y Centro de Educación Universal de la Institución Brookings.

Martínez, E.J, De la Torre, M.J. y Ruiz, A. (2017). Gamificación como nueva metodología para mejorar el rendimiento académico entre los jovenes: una estrategia desde las clases de Educación Física. Novos Tempos do Fitness-da teoria á prática. 79-84.

McLennan, N. y Thompson, J. (2015). Educación Física de Calidad: Guía para los responsables políticos. UNESCO.

Monguillot, M., Arévalo, C., Zurita, C. Almiral, L. y Guitert, M. (2015). Play the Game: gamificación y hábitos saludables en educación física/Play the Game: gamification and healthy habits in physical education. Apunts. Educació física i esports, (119), 71-79.

Morente, H.; Romance, A.R.; Gil, F.J. y Benítez, J. (2018). Programa de entrenamiento escolar gamificado para fomentar la actividad física saludable a través de una metodología innovadora de realidad aumentada (RA). Trances, 10 (1), 475-486.

Navarro, D., Martinez, R. y Pérez, I. J. (2017). El enigma de las 3 efes: Fortaleza, fidelidad y felicidad. Revista Española de Educación Física y Deportes, 419, 73-85.

Ortí, J. (2018). La gamificación en educación física. Desarrollo de la condición física a través de Kahoot. Tándem Didáctica de la Educación Física, (60), 58-61.

Pascuas, Y.S., Vargas, E.O. y Muñoz, J.I. (2017). Experiencias motivacionales gamificadas: una revisión sistemática de literatura. Innovación educativa (México, DF), 17(75), 63-80.

Pérez, I.J. y Rivera, E. (2017). Formar docentes, formar personas: análisis de los aprendizajes logrados por estudiantes universitarios desde una experiencia de gamificación. Signo y Pensamiento, 36(70), 112-129.

Pérez, I.J, Rivera, E., Delgado, M. (2017). Mejora de hábitos de vida saludables en alumnos universitarios mediante una propuesta de gamificación. Nutrición Hospitalaria, 34, 942-951.

Pérez, I.J., Rivera, E. y Trigueros, C. (2017). "La profecía de los elegidos": un ejemplo de gamificación aplicado a la docencia universitaria. Revista Internacional de Medicina y Ciencias de la Actividad Física y el Deporte 17(66), 243-260.

Quintero, L.E., Jiménez, F.J., y Area, M. (2018). Más allá del libro de texto. La gamificación mediada con TIC como alternativa de innovación en Educación Física. Retos, (34), 343-348.

Trillo, A.V., Palomares, J., Gonzáles, T. y De las Heras, E. (2018). PROYECTO MARVEF: EQUIPO DE SUPERHÉROESY SUPERHEROÍNAS. En actas del XI Congreso Internacional de Actividades Físicas Cooperativas. Avilés.

Zichermann, G. y Cunningham, C. (2011). Gamification by Desing: Implementing games Mechanics in Web and Mobile Apps Cambridge, MA: O'Reilly Media. 